\title{
Eléments de réflexion autour de la didactique de l'écriture/réécriture du FLE en contexte algérien
}

\section{The process of teaching writing following the Algerian curricula}

\author{
HAYAT DJAOUdi \\ Université de Tizi-Ouzou \\ djaoudihayat@yahoo.fr
}

\begin{abstract}
The teaching methods are evolving and being redesigned. Thus, the whole idea of the writing process, among scholars, is facing big criticism. In such academic climate, the hope is to bring the learning of this important subject into meeting grade level standards. The aim is, probably, to move away from the traditional teaching that is mainly based on strengthening the language abilities not, necessarily, the writing skills. These traditional practices have shown many limitations in terms of developing the appropriate writing skills needed to acquire a new language. Therefore, the need for a new approach is becoming an urgent challenge among educators.
\end{abstract}

Key words

Didactics, reading, writing, teaching practices.

\begin{abstract}
Resumen
Enseñar a escribir es un ejercicio real en negociaciones permanentes, ajustes y adaptaciones; Los métodos y técnicas deben ser construidos y reconstruidos. En este sentido, lo que se cuestiona es la concepción misma de la escritura escolar. En estas condiciones, para basar una didáctica de la escritura en bases serias, es importante no trabajar más en la nostalgia de los métodos tradicionales caracterizados por la ausencia de una teoría articulada entre "escribir" y "reescribir". Este silencio sobre el interés en estas actividades ha llevado a su marginación por parte de los estudiantes.
\end{abstract}

\section{Palabras clave}

didáctica, lectura, escritura, prácticas de enseñanza. 


\section{Introduction}

Aux cours des dernières années, les décideurs politiques ont mis en œuvre une série de réformes touchant les différents niveaux d'enseignement afin de pallier les insuffisances, favoriser l'expansion de la société éducative et faire face à une demande pressante de modernisation. Parallèlement, le développement de l'enseignement du français langue étrangère en Algérie est l'une des préoccupations majeures du système éducatif, eu égard aux modifications perpétuellement apportées dans les curricula. Mais à quel prix en matière d'efficacité?

Parler de curriculum signifie parler d'un optimum pédagogique, déterminé par des choix de politique éducative, à la fois objectifs de formation à atteindre, contenus disciplinaires à transmettre, stratégies didactiques à mettre en œuvre, instruments de contrôle et critères d'évaluation. (Gauthier, 2011).

En ce sens, la notion de curricula (pluriel de curriculum) renvoie à la programmation et l'organisation des activités d'enseignement/apprentissage. Ce plan d'actions éducatives structurées englobe l'ensemble des contenus sélectionnés, l'énoncé des finalités ciblées, les modalités d'interventions pédagogiques et d'évaluation des acquis.

L'analyse des contenus des programmes des trois cycles d'études, qui s'échelonnent du primaire au secondaire, nous permet dans une large mesure de dresser un diagnostic des pratiques en œuvre; c'est-à-dire de mieux comprendre les caractéristiques de l'enseignement/ apprentissage de l'écriture/réécriture. Plus exactement, il sera question de mettre l'accent sur ce que cet enseignement pense et privilégie et sur ce qu'il ne pense pas et ce qu'il exclut. Dans ce contexte, nombre d'interrogations fondamentales peuvent être soulevées, à savoir: Existe-il véritablement des pratiques scripturales notamment des productions écrites? Quelles en sont leurs limites? Et quelle formalisation didactique serait à construire?

Le présupposé qui guide notre analyse descriptive est le suivant: le développement des compétences scripturales chez les apprenants demande un changement de pratiques pédagogiques et didactiques. Pour ainsi dire, nous estimons que les programmes d'études, avant d'être le référentiel de compétences à acquérir, devraient dans un premier temps être un outil au service du changement. En ce regard-là, il ne s'agit pas uniquement de dresser la liste des compétences à faire acquérir aux apprenants, mais aussi et surtout de construire de nouvelles pratiques pédagogiques et didactiques qui permettraient justement l'acquisition de ces compétences.

\section{Méthodologie d'enseignement de l'écriture en Algérie}

Le modèle d'enseignement/apprentissage du français langue étrangère existant est fondé sur un enseignement répétitif et systématique du lexique, de la grammaire et de l'orthographe. La rédaction, souvent limitée à des reproductions de phrases collées les unes aux 
autres, est une activité qui est très fréquemment précédée de lecture et d'analyse de textes; cette pratique montre que l'écriture/lecture sont étroitement liées. Un autre pôle de l'enseignement/apprentissage de l'écriture est constitué de résumés de textes.

En explorant le contenu des programmes d'étude des différents cycles, notamment le moyen et le secondaire, nous remarquons une certaine diversité au niveau de la typologie textuelle. Cette typologie est souvent présentée sous forme de grille (dire, convaincre, expliquer, prescrire, décrire, etc.), ce qui permet aux enseignants de mieux classer les écrits et de mieux baliser les productions écrites des élèves. En analysant de près les curricula, il nous a été donné de constater que l'objectif assigné aux élèves consiste à reconnaître et à nommer les différents types de textes (descriptif, argumentatif, explicatif, etc.) et non de s'en servir pour mieux lire et surtout mieux écrire.

Nous constatons que l'accent est surtout mis sur l'analyse de ces types de textes et non sur la production effective et autonome des élèves. Dans cette optique, il importe plus d'arriver à reconnaître la dominante du texte afin de le classer que de produire un texte appartenant à tel ou tel type. A titre illustratif, voici quelques extraits tirés des programmes officiels du français au secondaire, et qui étayent davantage notre position:

L'élève est capable de distinguer le texte argumentatif des autres types de texte; retrouver à l'intérieur d'un texte argumentatif les énoncés narratifs, descriptifs, explicatifs; interpréter une image pour en dégager la visée argumentative; argumenter pour plaider une cause ou la discréditer. Les techniques d'expression: la prise de notes à partir de l'écrit ou de l'oral; exposer pour démontrer ou pour présenter un fait, une notion, un phénomène. Les techniques d'expression: la prise de notes à partir de l'écrit ou de l'oral (Ministère De L'éducation Nationale: 2005).

Toujours au secondaire, se limitant à la rédaction de résumés de textes, les élèves ne sont jamais confrontés à la dissertation, fait très surprenant et véritable inconvénient du développement des compétences à ne pas sous-estimer. Ceci constitue une réalité bien regrettable et explique pourquoi elle pose problème à un nombre d'étudiants au supérieur. Paradoxalement, l'entraînement à la dissertation en langue arabe est pratiqué, notamment dans les matières littéraires, à savoir la philosophie, la géographie et l'histoire; dans ce contexte, nous ne pouvons à proprement parler d'une didactique intégrée.

Nous évoquons ici l'articulation des modalités d'enseignement de l'écriture entre deux langues, en l'occurrence l'arabe et le français et ce, durant le cursus scolaire. Plus exactement, "il s'agit de relier les différentes voies d'accès aux langues, en construisant des curricula intégrés qui permettent d'articuler tout ce qui relève des approches plurielles des langues et des cultures" (Castellotti, 2006).

Dans cette perspective, Bruno Maurer met l'accent sur les apports de la didactique convergente dans les pratiques pédagogiques. D'après l'auteur, la pédagogie convergente se présente comme une "méthodologie des langues en milieu multilingue" (Maurer, 2013). 
Cette pédagogie est principalement axée sur l'apprenant et s'appuie sur des méthodes actives susceptibles de développer son autonomie. C'est une théorie d'apprentissage favorisant l'élaboration des hypothèses, son apport tient "à la convergence au plan de la méthodologie de l'enseignement de la L1 et de celui de la L2. Les deux langues sont enseignées selon les mêmes principes. C'est à ce niveau que se situe la convergence". (Maurer, 2013: 32).

Il importe de noter que cette nouvelle orientation favorise l'unification des enseignements sur le plan des stratégies et des supports; ce genre de pratique contribue dans une large mesure «au développement d'une pédagogie communicative plurilingue" (Castellotti, 2006: $320)$.

De surcroît, nous évoquons les travaux issus de la didactique du plurilinguisme. Dans cette optique, “on s'intéressera aussi bien aux travaux qui favorisent la transversalité des enseignements qu'à ceux qui visent à des modes d'alternance raisonnée des langues" (Candelier \& Castellotti, 2013).

Parallèlement, la grammaire n'aborde pas l'élaboration du texte et se limite uniquement à une description phrastique. Nous constatons, en effet, une carence dans la description des relations transphrastiques ainsi qu'une prédominance encore très générale de la grammaire de la phrase dans notre tradition pédagogique du français langue étrangère, même au niveau avancé (secondaire). Or, à ce stade d'apprentissage, il serait nécessaire et fort bénéfique de développer une grammaire textuelle pour pouvoir assurer le passage de la phrase au texte. Quant aux aspects liés à la communication, ils semblent ne pas être intégrés à l'enseignement, puisque l'objectif premier de l'écriture, qui est de communiquer, ne semble pas visé.

Apprendre aux élèves à rédiger des textes de différents types demande que l'on se penche sur la nature de ces textes pour mieux comprendre leurs caractéristiques. Force est de constater que le programme de français ne cesse de subir des réformes diverses; toutefois, l'enseignement de l'écriture n'est toujours pas bien implanté. Jusqu'à présent, les techniques d'expression écrites, centrées sur le processus de fabrication textuelle inspirées notamment du champ de la génétique textuelle, ne sont pas enseignées; l'essentiel est de développer les compétences linguistiques et non scripturales.

Il convient de souligner le caractère excessivement global des activités d'écriture qui sont habituellement pratiquées en classe, activités qui, de manière paradoxale, présument déjà acquises les compétences que les enseignants sont censés faire acquérir aux apprenants. L'écriture n'est finalement pas enseignée en tant que telle, elle se présente comme la synthèse des autres enseignements, essentiellement les sous-systèmes de la langue: orthographe, grammaire, vocabulaire, conjugaison, etc.

Cette absence d'enseignement de l'écriture s'articule, sans doute, à une absence de théories bien fondées sur l'écriture et son apprentissage. Dans ce cadre, l'écrit est également réduit par le temps qui lui est consacré dans les cours de français depuis le primaire jusqu'au 
secondaire. Il n'est pas étonnant, de ce fait, de constater que les élèves attribuent, de façon assez systématique, leur réussite ou leur échec au facteur de "don."

Ce qui vient d'être dit permet de se rendre compte à quel point les modes d'enseignement de l'écriture sont limités; ils nous semblent reposer sur un principe essentiel, celui de l'enseignement des sous-systèmes sans relation avec l'organisation textuelle des éléments (principes de cohérence et cohésion).

Par ailleurs, les programmes officiels passent sous silence la réécriture; il ressort que cette pratique est délaissée et ne constitue nullement une préoccupation première. Nous estimons qu'il est urgent d'outrepasser ces représentations et intégrer un enseignement optimal et régulier de la réécriture qui est au cœur des débats didactiques actuels. Dès lors, il importe de suggérer quelques pistes d'intervention visant à légitimer cette opération reléguée bien souvent au second plan.

L'examen des documents officiels et des programmes relatifs à l'enseignement de l'écriture en Algérie nous a permis de constater que les opérations d'écriture-réécriture n'occupent pas de place apparente dans les activités scripturales envisagées en classe (voir le site du Ministère De l'Education Nationale, "programmes de français au moyen en contexte algérien", "programmes de français au secondaire en contexte algérien"). Cette marginalisation et ce silence doivent impérativement être portés sur le devant de la scène didactique.

Ces pratiques ont manifesté quelques limites et ont fait ressentir la nécessité de transformations importantes auprès des responsables et des chercheurs en didactique. Néanmoins, il n'existe bien sûr pas un modèle de rechange tout prêt: d'abord parce que l'essor des recherches en didactique a entraîné une méfiance devant toute pensée dogmatique. Ensuite, parce que les recherches en écriture sont en réalité fort récentes.

L'écriture n'est pas en tant que telle une discipline d'enseignement et pourtant elle intervient dans toutes les disciplines et demeure un mode d'accès, privilégié sinon exclusif, aux connaissances et à la certification. Dans ce qui suit, nous tenterons de construire une formalisation à vocation heuristique de l'écriture qui nous paraît être intéressante pour la didactique de l'écriture en fonction des connaissances théoriques disponibles à l'heure actuelle.

\section{La question de littéracie}

Force est de préciser que la "littéracie" a progressivement intégré les travaux de recherche en didactique et sciences de l'éducation. Sur le plan étymologique, ce terme recouvre la maîtrise des différentes facettes de l'écrit, son rapport demeure problématique avec la maîtrise de l'oral.

A vrai dire, cette notion rend compte de la complexité de l'enseignement/apprentissage de la lecture-écriture et interroge les pratiques et acquis des scripteurs-lecteurs en contexte (linguistique, culturel, social, ....), comme le souligne L. Kadi "les pratiques et 
usages varient selon les langues et les cultures, selon le temps et selon l'espace" (Kadi, 2009). Pour ainsi dire, le champ de la littéracie est articulé autour de recherches dont les résultats et les mises en perspectives permettent de repenser leurs modalités d'enseignement/apprentissage et mieux fonder les démarches de leur transmission aux scripteurs-lecteurs. Pour citer C. Barre-De Miniac, "les recherches se situent maintenant en amont et en aval des premiers apprentissages de la lecture et de l'écriture" (Barre-De Miniac: 2003).

L'enjeu majeur est de mieux cerner cette complexité et mettre en évidence l'articulation des usages privés et sociaux de l'écrit, des pratiques scolaires et sociales et ce, dans une perspective théorique pluridimensionnelle (dimension langagière, cognitive, sociale, culturelle...).

La littéracie est une notion heuristique qui incite à multiplier les interactions et les activités entre "lecture", "oral" et "écriture". Elle a fait l'objet de définitions multiples, retenons celle de J-P. Jaffre qui semble tout à fait pertinente. Selon lui, le terme désigne:

l'ensemble des activités humaines qui impliquent l'usage de l'écriture en réception et en production. Elle met un ensemble de compétences de base, linguistiques et graphiques, au service de pratiques, qu'elles soient techniques, cognitives, sociales ou culturelles. Son contexte fonctionnel peut varier d'un pays à l'autre, d'une culture à l'autre, et aussi dans le temps. (cité par Barre-De Miniac et al.: 2007).

De nos jours, il est indéniable que la question du développement des compétences scripturales représente une problématique centrale dans les recherches en didactique et constitue un véritable enjeu dans les systèmes éducatifs.

Par ailleurs, les compétences langagières en général constituent un vaste champ d'investigation, elles recouvrent à la fois la compréhension orale/écrite (processus langagiers réceptifs) et l'expression orale/écrite (processus langagiers productifs). L'enseignement des langues s'articule bien autour de ce découpage. Ces quatre habiletés, même si elles sont fortement corrélées, sont considérées comme distinctes puisqu'elles peuvent se développer inégalement.

Il convient de préciser que l'oral et l'écrit constituent deux entités imbriquées et complémentaires, on ne peut concevoir l'une sans l'autre. Cependant, en l'état actuel l'oral est “considéré comme insaisissable, éphémère, il bénéficie d'une image négative par rapport à l'écrit, auquel il est constamment comparé et auquel il sert le plus souvent de support" (Alrabadi, 2011). En ce sens, on recourt bien souvent à l'oralité à travers les séances de lecture, ces dernières servent de passerelle à l'apprentissage de l'écriture.

Valorisé culturellement, l'écrit véhicule un concentré de toutes les normes, qu'elles soient lexico-syntaxiques, orthographiques, stylistiques, ou encore relatives à la planification du texte et à la gestion transphrastique de l'information dont il est le support.

Il est indéniable que toute situation d'écriture nécessite de la part du scripteur une mo- 
bilisation simultanée de l'ensemble de ses compétences linguistiques, en même temps que le contrôle de toutes sortes d'opérations locales et globales de mise en texte.

En outre, l'écriture est parmi tous les actes langagiers (écouter, parler, lire, écrire), celui qui nécessite le plus long temps d'apprentissage en raison de sa complexité.

Dans ce sillage, J-P Robert précise qu'“en didactique des langues, l'écrit fait partie de ces notions jumelées dont l'étude est incontournable: écrit/oral, lecture/écriture, compréhension/production, phonème/graphème, etc.”(Robert, 2008: 36 ).

Comme nous l'avons déjà précisé, la pratique de lecture-écriture est dominante notamment au moyen et au secondaire. C'est une pratique qui consiste à utiliser des données à travers une observation rigoureuse des textes, activité selon laquelle se fait l'initiation à la lecture d'une variété textuelle. Cette relation lecture-écriture est la véritable armature de la séquence didactique, ces deux concepts sont tellement liés qu'on n'arrive plus à concevoir l'un sans l'autre. Il est à noter qu'en pratique, l'adoption d'une démarche fondée sur une simple transposition entre "lire" et "écrire" conduit les apprenants à secondariser l'activité d'écriture et à établir des corrélations constitutives entre ces deux modes d'accès au texte.

Cette pratique risque de rendre l'activité d'écriture comme une simple transposition du travail de lecture du texte source. En ce sens, l'objectif assigné devient de "reconnaître et de nommer les types [...] l'enjeu ne saurait être de s'en servir comme des instruments d'étiquetage [...] leur grand intérêt est de mettre en lumière des régularités organisationnelles dans la formation des textes" (Reuter, 1996: 120).

Il serait en effet intéressant de guider les opérations scripturales des apprenants et mettre en évidence les techniques de génération textuelle, à savoir les opérations de planification, de mise en texte et de révision afin de construire une image claire du produit final. De façon plus spécifique, il importe de renforcer davantage les activités relatives à la production des différents types de textes qui caractérisent la langue française. Dès lors, il devient nécessaire de leur faire travailler chacun des types d'une manière systématique et surtout progressive, de sorte que les textes produits soient de plus en plus longs au cours des différents cycles.

Au plan des méthodologies d'enseignement, nous soulignons une centration majeure sur les genres que sur l'activité d'écriture, cette démarche devrait être bannie et controversée dans l'avenir immédiat. La primauté de la finalité pratique des activités d'écriture devrait impérativement être affirmée, non seulement dans la configuration des programmes mais aussi et surtout dans les pratiques effectives. Un important travail reste à accomplir, ce qui exigerait une nouvelle conceptualisation didactique en ce domaine spécifique de l'écrit. Comme développée ci-dessous, cette conceptualisation se caractérise principalement par un changement des habitudes scolaires liées à la planification, mise en texte, révision et réécriture.

Par ailleurs, il importe toujours de laisser une place à la créativité. Dans cette optique, l'écriture doit être enseignée comme une expérience personnelle et une pratique spontanée. 
Les consignes devraient ainsi laisser la liberté aux apprenants de choisir eux-mêmes les objets de leurs discours; cette approche devrait favoriser une plus grande motivation.

Pour commencer à cerner notre formalisation didactique, nous insistons sur un point crucial: les textes d'écrivains ne devraient plus être perçus comme des textes génies à vénérer mais doivent être considérés comme de simples instruments de travail. Il est à signaler que ce centrage sur des textes originaux, achevés et valorisés nourrit davantage la conception de l'écriture comme don. Dès lors, un paradoxe surgit: “ comment imiter ce qui est posé comme inimitable? Ou encore puisque le travail sur ces textes repose sur une valorisation et une imprégnation -transformant la référence en révérence- comment utiliser et s'approprier ce dont on a occulté la fabrique"(Barre-De Miniac, 1996: 20).

Le rapport à ces référents doit impérativement changer; il ne faudrait pas lire ces auteurs pour les vénérer mais plutôt pour s'y référer afin de puiser des techniques procédurales en matière d'écriture. Nous conviendrons que ces pratiques qui perdurent encore ne sont pas susceptibles d'aider les apprenants en difficulté; elles sont révélatrices de l'absence de l'enseignement de l'écriture en tant que discipline à part entière.

\section{Renoncer aux méthodes traditionnelles}

La réflexion pédagogique se nourrit largement de ces avancées pluridisciplinaires, passant d'approche centrée sur les méthodes de lecture-écriture à une problématique plus large de la maîtrise de la langue pour un accès à la culture de l'écrit. (Barre-De Miniac, 1996: 12).

Dès lors, une problématique majeure est soulevée, celle de la conception de l'écrit comme produit des activités de langue. Nous nous rendons compte que l'enseignement traditionnel présente des points faibles; il semble reposer sur l'imitation de textes modèles ou l'appropriation des normes grammaticales.

Dans ce qui suit, nous nous permettons de fonder une critique à l'encontre des méthodes traditionnelles qui perdurent encore dans les pratiques didactiques et qui se focalisent essentiellement sur l'écrit et non sur l'écriture. Nous insistons d'abord sur la nécessité de renoncer aux méthodes dites traditionnelles, centrées sur des points de grammaire ou le bon usage pour développer les compétences communicatives à l'écrit. Il faut reconnaître que cette génération d'exercices et ces pratiques désuètes et artificielles n'étaient guère des conditions propices à un véritable apprentissage de l'écriture.

Un regard sur les contenus des programmes nous permet de constater que l'écrit a souvent été considéré comme un ensemble de sous-savoirs à faire acquérir, selon un modèle hiérarchique, en commençant par l'enseignement des règles grammaticales et orthographiques. Une telle démarche serait à bannir, elle se traduirait inévitablement par une production très contraignante dans la mesure où "la maîtrise de certaines règles ne conduiraient pas 
forcément à l'application automatique de ces mêmes règles en situation d'écriture" (Cornaire \& Raymond, 1994: 89).

Pour posséder une compétence scripturale, il est indispensable que les procédures de base qui y sont impliquées aient été automatisées; cela exige évidemment des exercices spécifiques et un renforcement de pratiques axées sur les activités d'écriture (créatives et spontanées), de révision (avec buts spécifiques) et de réécriture (ajout, remplacement, suppression, déplacement).

Cependant, des cours de grammaire, de vocabulaire et d'orthographe ne sauraient à eux seuls tenir lieu d'enseignement de l'écriture; ils servent uniquement de supports et ne peuvent éclairer les mécanismes de l'écriture, comme le mentionne J.-f. Halte: “que l'écrit ne soit pas la résultante mécanique de l'apprentissage de l'orthographe, des temps verbaux et du complément d'objet direct" (cité par Barre De Miniac, 1996: 52).

Il ressort que l'écriture ne se présente pas comme une synthèse des autres enseignements relatifs aux sous-systèmes de la langue, mais doit être enseignée en tant que discipline à part entière. Une formalisation didactique de l'écriture est à construire, à la lumière de la réflexion d'Yves Reuter qui soutient que "faute de théorie suffisante du texte, de l'écriture, du développement des compétences et de l'apprentissage, les interventions enseignantes et les constructions didactiques restent du bricolage, y compris dans leur évaluation" (Barre De Miniac, 1996: 66).

Il serait bon que cette situation qui perdure change, si nous voulons voir les compétences scripturales des élèves se développer (du mot à la phrase, de la phrase à l'énoncé, de l'énoncé au texte) dans le sens du discours qui recèle des compétences communicatives.

\section{Articulation entre théorie et pratique}

Nous pouvons avancer que la théorie et la pratique scripturales sont deux phases nécessaires, emboitées et complémentaires; nous ne saurons, par conséquent, considérer l'une sans l'autre. Autrement dit, "l'écriture et la mise en œuvre de son acquisition supposent une interpénétration, une porosité entre sa pratique et sa théorisation” (Reuter, 1996: 111 ).

Au lieu de se succéder, elles doivent à notre sens s'engendrer mutuellement; d'où l'intérêt des activités d'improvisation dans l'enseignement de l'écriture tel que nous le concevons et le préconisons (écriture poétique, narration à partir de supports visuels, exercices de style, association d'idées, dictées, etc.). Ainsi, selon une approche formative, et en fonction des besoins des apprenants, l'enseignant peut réaliser en classe des activités non programmées au préalable. Dans cette optique, le discours théorique et le faire scriptural ne correspondraient pas à un clivage mais constituent plutôt un continuum, c'est-à-dire un dispositif cohérent et articulé.

Force est de préciser qu'une démystification de l'écrit et de l'écriture permettrait aux 
apprenants d'être en sécurité linguistique et repenser leurs écrits en termes de production et d'expression. En outre, nous estimons que la fréquentation précoce et éclairée de la culture écrite conduit à instaurer un rapport positif aux savoirs linguistiques sous-jacents.

Dans une perspective socio-didactique de l'écriture, nous ne pouvons négliger l'incidence des paramètres de différents ordres qui interviennent dans cette activité: ceux d'ordre socioculturel qui se rapporte à ce qui a été préalablement acquis par les apprenants, grâce à la fréquentation des écrits dans leur contexte d'appartenance, et aux représentations qui y circulent à propos de l'acte d'écriture en lui-même.

Il convient de noter que la formalisation théorique est loin d'être évidente; elle n'est d'ailleurs pas facile à construire de manière précise et exhaustive, dans la mesure où elle doit répondre aux besoins des apprenants; néanmoins, une formalisation plausible serait à construire en cours de route par l'enseignant. C'est dire, tout simplement, rompre avec les méthodes dites traditionnelles dans lesquelles le cours précède toujours l'application; il est d'ailleurs vain de vouloir l'appliquer systématiquement dans l'enseignement de l'écriture.

Dans cette perspective,

il serait intéressant de construire un processus plus dynamique: émergence des problèmes individuels, transformation de ces problèmes en problèmes collectifs, susceptibles d'être traités dans des situations de théorisation théorique, transformation de cette formalisation en instrument d'aide à l'écriture. (Reuter, 1996: 116 ).

Afin de faciliter les transferts, la logique des apprentissages telle que nous la préconisons devrait privilégier une diversification des modalités de fonctionnement; les phases peuvent ainsi se présenter dans des ordres différents. En fonction des attentes des apprenants, l'enseignement de l'écriture doit fonctionner avec des allers et retours entre théorisation et mise en pratique. L'enjeu est double: en ce sens, nous pouvons d'une part appliquer une réflexion théorique et d'autre part théoriser des questions pratiques.

\section{Changer les habitudes scolaires liées à la mise en texte}

"Mettre tout un livre dans une page, toute une page dans une phrase et cette phrase dans un mot $[\ldots]$ Condenser, synthétiser, aller à l'essentiel; voilà une bonne matière à concours" (Gilder, 2009:119).

Que cette ambition d'économie verbale puisse guider tout apprenant. Nombre d'épreuves y pourvoient telles que le résumé et la synthèse de textes. Ce type d'activités, systématiquement pratiqué, oblige l'apprenant à être sobre, à économiser les mots, bref à être concis.

Il importe de poser et d'inculquer, dès les premières années d'apprentissage, cette règle: une idée, une phrase offrant un sens complet, et amener les apprenants à tronçonner les 
longues phrases, éviter les phrases labyrinthes et enchevêtrées qui conduiraient à des contorsions de style. Cependant, à un stade d'enseignement/ apprentissage de l'écriture plus avancé, il convient de développer chez les apprenants la compétence d'expansion verbale.

Parallèlement, afin de développer la capacité à structurer, il convient de proposer des activités de rédaction d'articles à partir de prises de notes proposées par l'enseignant; ou encore proposer des textes-puzzles et demander de les reconstituer. L'objectif de ce type d'activités serait de créer des enchaînements, soigner les reprises lexicales et explorer les phénomènes de l'organisation textuelle.

Il serait également intéressant de leur proposer d'améliorer la cohérence d'un texte en supprimant les redondances; l'apprenant sera ainsi amené à mobiliser toutes ses ressources anaphoriques. Ou encore développer une pratique consistant à réécrire par substitution; l'objectif de cette activité est de garder la structure syntaxique d'un texte en remplaçant un mot par des synonymes appartenant à la même catégorie grammaticale. Ce genre de pratique constitue un entraînement pour l'usage des procédés anaphoriques et permet à l'apprenant d'éviter toutes sortes de réitérations.

Par ailleurs, il est important de faire observer aux apprenants comment s'opère l'équilibrage entre la nécessité d'apporter constamment de l'information nouvelle, et de référer sous forme d'anaphores à des éléments déjà cités dans les énoncés antérieurs. Pour mieux guider les apprenants dans leur compréhension des règles de cohérence, il est fondamental de faire l'analyse des thèmes et des rhèmes de textes proposés à la lecture ainsi que les différentes progressions thématiques (thème constant, linéaire et éclaté).

La cohérence textuelle répond aux quatre règles bien spécifiques à savoir la continuité, la non contradiction, la progression et la relation. Selon notre point de vue, lors de la correction des copies, l'enseignant devrait préciser le principe de cohérence violé par l'apprenant, et ne pas se contenter de mentionner "incohérent" qui a plutôt un sens vague et indéterminé. Nous estimons que cette absence de précision et le recours à ce type d'annotation ne permet ni de comprendre la nature du problème ni l'amélioration de l'écrit. En matière de cohérence, il est capital de pointer et d'expliciter la nature du dysfonctionnement pour que l'apprenant puisse situer ses lacunes et du coup éviter de les reproduire ultérieurement.

\section{La réécriture: un dispositif du développement des compétences scripturales}

"La réécriture se trouve radicalement exclue des pratiques traditionnelles liées à la rédaction" (Reuter, 1996: 19). Si elle a conquis une place importante ces dernières années, c'est en fonction des recherches qui ont montré qu'"écrire" c'est "réécrire", c'est entrer dans une dynamique scripturale, c'est se représenter son texte comme transformable, c'est tout simplement prendre de la distance. Un domaine de recherche qui ne saurait à l'heure actuelle être oublié, si nous voudrions vraiment comprendre l'écriture, est celui de la génétique textuelle. 
Cette discipline jeune constitue l'une des principales innovations critiques, elle s'apparente à l'étude des brouillons d'écrivains dans une perspective diachronique. En prenant appui des dossiers génétiques ou des états intermédiaires, le généticien tente de reconstituer les phases de réécriture ainsi que le cheminement créatif de l'auteur. Autrement dit, l'étude des marques scripturales constituées des annotations, biffures et ratures permettent d'appréhender la genèse de l'œuvre.

Après avoir examiné de près le contenu des programmes scolaires, nous avons relevé une nette marginalisation des activités liées à la réécriture. Partant de ce constat, la réécriture doit désormais constituer un objectif et un outil d'enseignement et d'intervention pédagogique et du coup faire l'objet d'une réforme, dans la mesure où apprendre à écrire implique nécessairement apprendre à réécrire. Dans cette perspective, "écrire" et "réécrire" s'analysent comme deux aspects qui relèvent d'une même activité.

"Réécrire est une situation paradoxale et inconfortable"(Delmotte \& Penloup, 1994). C'est une pratique difficile à manier car elle est complexe en soi; elle peut être envisagée à travers des textes que produit l'apprenant-scripteur lui-même, ou encore comme une reprise de textes écrits par ses pairs en y jetant un regard critique.

Il conviendrait donc de développer l'écriture en projet; les didacticiens parlent également d'ateliers ou de chantiers d'écriture. Un chantier est "un travail dans la durée, il comporte de nombreuses séances, chaque fois ciblées avec précision et non interchangeables. Selon le type de texte travaillé et selon les disponibilités et les urgences des classes" (Vallegeas, 2012). Ces séances peuvent s'étaler dans le temps sur une semaine, un mois ou encore sur tout un trimestre.

La primauté de la finalité pratique des activités d'écriture/réécriture devrait impérativement être affirmée non seulement dans la configuration des programmes mais aussi et surtout dans les pratiques effectives. Dès les premières années d'apprentissage, il importe d'aider les apprenants à “admettre que faire un brouillon n'est pas déshonorant, que réécrire n'est pas dévalorisant” (Szajda-Boulanger, 2005: 531). L'objectif escompté est de les guider dans l'activité de révision, de les initier progressivement à la réécriture et de leur montrer que la rature n'est pas une marque d'incompétence.

Plus exactement, il serait intéressant de cibler des points précis, de centrer leur attention sur des zones problématiques, de spécifier les mécanismes de réécriture et d'appréhender les limites d'une écriture non retravaillée.

Il nous été donné de constater que la tradition scolaire focalise généralement l'attention des apprenants sur la lecture et l'analyse de textes achevés et valorisés. Ce qui risque d'évacuer toute réflexion liée à leur fabrique, contribuer à conforter des représentations obstacles, nourrir le sentiment d'insécurité scripturale, et du coup bloquer l'écriture. 


\section{L'évaluation et l'auto-évaluation}

Nous évoquons principalement les avancées en matière de portfolio et de l'ensemble des initiatives qui amènent l'apprenant à prendre en main sa formation dans une logique contractuelle, interactive et collaborative.

Dans ce contexte, l'enseignant se met dans une posture de contrôleur et d'accompagnateur, l'apprenant quant à lui est un auto-évaluateur, un constructeur de savoirs au profit d'une démarche métacognitive de validation des acquis. Cette piste nécessite la mise en place d'une culture de responsabilisation et d'individualisation de la relation pédagogique.

L'apprenant est ainsi engagé à évaluer sa propre production en se servant d'un référentiel externe (grille, dictionnaire, etc.). Cette modalité peut favoriser sa réflexion sur le fonctionnement de ses productions et stimuler la structuration de ses conduites d'auto- évaluation.

Des interventions didactiques spécifiques seraient à effectuer si nous voulons amener les apprenants à bien écrire. Il faut d'abord les amener à identifier, analyser les problèmes auxquels ils sont confrontés et à affronter puis coordonner les points de vue.

Procéder, par exemple, à l'évaluation des copies en groupe est une autre activité que nous préconisons dès les premières années d'apprentissage. Dans cette optique, l'enseignant est amené à travailler progressivement en collaboration avec ses élèves qui en tireraient grand profit.

L'évaluation collective présente un double avantage. D'une part, à travers une approche réflexive, elle procure à l'apprenant de vrais lecteurs et développe en lui le plaisir de réagir et d'analyser les réactions d'autrui. D'autre part, elle présente une occasion pour le sujet-scripteur d'expliquer les choix qu'il a effectués, et c'est à l'enseignant ensuite de le guider et de lui expliquer l'origine de ses problèmes.

En nous inspirant du modèle de Hayes et Flower, nous préconisons, par ailleurs, un protocole de verbalisation à "haute voix" au cours de l'activité scripturale qui consiste à "demander à un sujet de réaliser une tâche en commentant ce qu'il fait " (Reuter, 1996:40). Cette pratique offrirait une procédure efficace et un intérêt pédagogique indéniable, dans la mesure où elle permettrait d'observer de près la manière dont les scripteurs-réviseurs explicitent les problèmes perçus et reconstruisent leur texte. Ces pratiques impliquent nécessairement une réduction de la taille des groupes des apprenants par classe, condition sine qua non pour garantir efficacité et faisabilité sur terrain.

Désormais, il est important d'installer de nouveaux dispositifs qui susciteraient questionnement et réflexivité nécessaires au travail de révision-réécriture. Une des tâches assignées à l'enseignant serait d'inciter l'apprenant à relire ses productions plusieurs fois en se fixant à chaque lecture un but bien précis et à les reprendre en produisant plusieurs versions. 


\section{Composantes linguistiques et textuelles}

"La nécessité de hiérarchiser différents plans didactiques amène enseignants et élèves à opposer des apprentissages de fait complémentaires, voire insécables" (David, 1994). Plus exactement, ces plans recouvrent des aspects imbriqués relevant d'une structure formelle et d'une structure profonde.

Il semble important de réévaluer singulièrement la place des composantes linguistiques et textuelles; inutile de scinder ces compétences scripturales qui ne peuvent être conduites que conjointement. Une des démarches préconisées serait de conduire les apprenants, tout au long des cursus scolaire et universitaire, vers une approche complémentaire des problèmes de mise en mots et de mise en texte, en ne différant ni les premiers ni les seconds.

A vrai dire, nous sommes quelques peu réservée à l'égard de la conception dominante qui dissocie les composantes de l'écriture et qui évacue bien souvent les compétences textuelles du savoir-écrire, supposant que seules les compétences linguistiques sont dignes d'être retravaillées.

L'expérience montre que, lors du travail de réécriture, la correction linguistique notamment orthographique est généralement bien mise en évidence, elle occupe une place centrale dans l'ensemble des réaménagements introduits par les apprenants. Néanmoins, il est essentiel d'analyser également les dysfonctionnements en termes de rapports, de leur donner du sens dans le cadre du mot, du syntagme ou de la phrase, comme dans l'organisation textuelle.

Force est de reconnaître que tout texte est susceptible d'être amélioré; l'intérêt du travail de réécriture est évident. Certes, la réécriture est un enseignement très contraignant, dans la mesure où cette pratique ne se réduirait pas à une simple correction superficielle essentiellement orthographique. Elle demande beaucoup d'effort, du temps et surtout une bonne gestion des ressources langagières et de mise en texte.

Pour surmonter ces problèmes, les enseignants peuvent envisager des ateliers d'écriture et de réécriture privilégiant les aspects textuels. Ces ateliers peuvent s'étendre sur un mois ou un trimestre, marquant le déroulement de l'activité scripturale en séquences et étapes incontournables. Plus précisément, il s'agirait de retravailler les écrits "par un retour sur les aspects discursifs, textuels et stylistiques, en reportant à une étape ultérieure la gestion de la ponctuation et de l'orthographe" (Paradis, 2013). Dans ce contexte, enseignants et apprenants adoptent une posture de "lecteurs" et non de " correcteurs".

Nous estimons qu'il conviendrait de réévaluer la place réservée à l'enseignement des composantes linguistiques, en leur consacrant un temps d'apprentissage qui ne soit ni excessif ni minoré. Nous devons, dès lors, penser des apprentissages qui permettent aux apprenants de saisir le fonctionnement de la langue par l'écriture et non les usages séparés de l'une et de l'autre. 
Il est clair que la réécriture représente une tâche conséquente aussi bien pour les enseignants que pour les apprenants. Il s'avère en effet difficile de retravailler les productions à tous les niveaux; c'est pourquoi, l'enseignant doit donner une finalité à la réécriture et opérer un choix des critères les plus pertinents qu'il importe de retravailler prioritairement.

Une des modalités didactiques que nous préconisons consiste à fournir aux apprenants un guide de relecture grâce auquel ils pourront travailler et retravailler leurs textes pas à pas. Il est utile voire nécessaire de communiquer une grille de relecture/réécriture comportant des critères explicites, pertinents et bien précis; elle représente une référence des attentes et un reflet d'une transparence didactique. En s'y référant, les apprenants focaliseront leur attention sur des points cruciaux qui doivent être réécrits. Dans cette perspective, toute démarche de réécriture doit impérativement être guidée et accompagnée.

\section{Conclusion}

L'écriture envisagée dans la formation scolaire constitue un champ privilégié visant le développement des compétences scripturales. Une approche de ce type suppose la remise en question des modes de conception de l'écriture (cadre conceptuel, matériaux d'enseignement, dispositifs d'évaluation, réorganisation des programmes...), ainsi qu'une prise en considération des avancées issues des disciplines de référence, essentiellement la didactique des langues et les Sciences de l'éducation.

Force est de constater que l'enseignement de l'écriture en Algérie a sans doute besoin d'un souffle de renouveau; il doit être soumis à une réflexion qui tienne compte de la méthodologie en vigueur à savoir l'approche par les compétences et l'orientation actionnelle. Autrement dit, nous pensons qu'un changement de perception de ce que représente l'acte d'écriture pourrait contribuer à redonner une place plus stimulante à l'enseignement de l'écriture. A cet effet, la coordination d'un nombre de paramètres d'ordre situationnel, procédural et méthodologique doit être réalisée et concrétisée sur terrain.

L'activité scripturale doit, à notre sens, être l'objet d'un apprentissage régulier et progressif; cependant, sur terrain et en pratique, la réalité est toute autre. Dès lors, il convient de récuser définitivement les démarches d'enseignement antérieures, installer dans l'urgence et concrètement une configuration didactique moderne qui se traduit par une adaptation des contenus et des méthodes d'enseignement.

Amener les apprenants à devenir sujets de leurs écrits, fournir des outils théoriques et méthodologiques actualisés, adapter les populations scolaires à des stratégies scripturales à la fois souples et efficaces constituent des aspects pertinents dont il conviendrait de tenir compte dans une didactique de l'écriture. Il est donc temps de promouvoir de nouvelles pratiques et techniques de facilitation procédurale de l'écriture, visant à polariser l'effort de l'apprenant sur les problèmes de mise en texte. 
Par ailleurs, pour accroître l'efficacité de l'enseignement de l'écriture, nous considérons que la formation des formateurs est une piste qui nécessite une concrétisation immédiate et ce, dans les différents cycles d'enseignement. Il serait en effet souhaitable de prendre en charge et d'aider spécifiquement les jeunes futurs enseignants à combler leurs propres lacunes et les amener à aborder des démarches d'enseignement d'écriture complexes, efficaces et adaptables.

La formation universitaire des futurs enseignants devrait se focaliser principalement sur des démarches visant la maîtrise de savoirs théoriques, de notions et de normes. Parallèlement, elle devrait accorder une place centrale à l'objectif épistémique et aux aspects praxéologiques relatifs aux savoir-faire pratiques. Cette démarche de formation paraît bien efficace, l'enjeu essentiel de la formation "se situe dans l'articulation efficace de ces deux dimensions." (Bronckart, 2011).

Autant de réflexions, de moments d'arrêts des limites des pratiques installées et de voies ouvertes pour un éventuel renouvellement des pratiques didactiques en matière d'enseignement-apprentissage de l'écriture/réécriture. Néanmoins, la didactique de l'écriture est encore en suspens; elle est à ses premiers pas comme le souligne Yves Reuter: “nous n'en sommes qu'à la préhistoire de la didactique de l'écriture parce que l'écriture en tant que telle n’est pas enseignée" (cité par Barré De Miniac, 1996: 49).

\section{Références bibliographiques}

Alrabadi, Elie. 2011. “Quelle méthodologie faut-il adopter l'enseignement/apprentissage de l'oral?" in Revue de Didactique, Langue et Littérature, ${ }^{\circ} 23,15-34$, [consulté le 31/01/2018]. <https://revistas.ucm.es/index.php/DIDA/article/viewFile/36308/35153>.

BARRE-DE MiniaC, Christine.1996.Vers une didactique de l'écriture, pour une approche pluridisciplinaire. Bruxelles, De Boeck.

Barre-De Miniac, Christine. 2003. "Savoir lire et écrire dans une société donnée" in Revue Française de Linguistique appliquée, volume 8, IUFM de Grenoble, 107-120, [consulté le 31/01/2018]. < https://www.cairn.info/revue-francaise-de-linguistique-appliquee-2003-1page-107.htm $>$.

Barre-De Miniac, Christine, Catherine, Brissaud \& Marielle, Rispail. 2007. "La littéracie. Conceptions théoriques et pratiques d'enseignement de la lecture-écriture" in Repères, $\mathrm{n}^{\circ}$ 36, 263-272, [consulté le 31/01/2018], <http://journals.openedition.org/reperes/492>.

Bronckart, Jean-Paul. 2011. "Un demi-siècle de didactique de l'écrit dans les pays francophones: bilan et perspectives" [consulté le 05/05/2018]: < http://periodicos.pucminas.br/ index.php/scripta/article/viewFile/4297/4442. >

Candelier, Michel. \& Véronique, Castellotti. 2013. "Didactique(s) du (des) plurilinguisme(s)" in Sociolinguistique du contact, Dictionnaires des termes et concepts, 179 -221 [consulté le 20/05/2017] <http://www.projetpluri-1.org/publis/Candelier\%20\&\%20Castelotti $\% 20>$. 
Castellotti, Véronique. 2006. "Une conception plurielle et intégrée de l'enseignement des langues - principes, modalités, perspectives" in Les Cahiers de l'Acedle, n 2, 319 -331, Université François Rabelais, [consulté le 05/03/2017], <http://acedle.org/IMG/pdf/Castellotti-V_cah2.pdf $>$.

Cornaire, Claudette \& Patricia, Mary Raymond. 1994. La production écrite, CEC, Canada.

DAVID, Jacques.1994. "Écrire, c'est réécrire. De la pertinence des ratures chez l'écolier” in Le Français Aujourd'hui, n 108, 6-18.

Delmotte-Legrand, Régine \& Marie-Claude, Penloup. 1994, "Du journal de bord au collège comme lieu d'émergence des représentations de la réécriture" in Repères, $\mathrm{n}^{\circ} 10$, Université de Rouen, 83-94.

GaUthiER, Roger-François \& Luisa, Lombardi.2011 "Modèles de curricula et politiques curriculaires en Italie", in Revue Internationale d'Education de Sèvre, n ${ }^{\circ}$ 56,41 - 50, [consulté le 21/02/2018] < http://journals.openedition.org/ries/1043>.

GILDER, Alfred. 2009. Le français administratif. Ecrire pour être lu, Edition Glyphe, Paris.

KADI, Latifa. 2009. "De la littéracie et des contextes” in Synergie, n6, 11-17 [consulté le 31/01/2018]<https://gerflint.fr/Base/Algerie6/kadi.pdf>.

MARER, Bruno. 2013. “De la pédagogie convergente à l'éducation bilingue: généralisation des langues nationales au Mali et transformations du modèle de la PC" in Education et société plurilingue, $\mathrm{n}^{\circ} 34,29-42$, [consulté le 20/05/2017] < www.cebip.com/download.asp?file=/ elementi/www/esp034>.

Ministere de L'educhtion Nationale. 2005. "Le nouveau programme de français de la 1ère année secondaire",01-09, [consulté le 10/02/2017]. < http://www.oasisfle.com/documents/nouveauprogramme_de_francais_1ere_annee_secon daire.htm $>$.

Ministere de L'education Nationale. 2005. "Programme de 2ème A.S, tableau synoptique", 01 -15, [consulté le 10/02/2017] < https://fr.scribd.com/doc/186718724/ProgrammeFr-de-La-2eme-As $>$.

PARADIs, Hélène.2013. "La réécriture" in Correspondance, volume 18, n³, 01-06, [consulté le 10/05/2017] <http://correspo.ccdmd.qc.ca/Corr18- 3/7.html>.

ReUter, Yves.1996. Enseigner et apprendre à écrire. Paris, ESF éditeur.

Robert, Jean-Pierre. 2008. Dictionnaire pratique de didactique du FLE. Paris, L'Essentiel.

SzaJda-Boulanger, Liliane. 2005. Ecriture et rapport à l'écriture des élèves de SEGPA, Thèse de Doctorat, université Lille III.

VAllegeas, A. (2012). “La réécriture au cycle3: représentations-pratiques-propositions didactiques" [consulté le 10/05/2018] < http://dumas.ccsd.cnrs.fr/docs/00/75/71/51/PDF/ download.php3.pdf> 
